\title{
Macroscopic description of capillary transport of liquid and gas in unsaturated porous materials
}

\author{
Mieczysław Cieszko
}

Received: 29 January 2015/Accepted: 12 January 2016/Published online: 22 January 2016

(C) The Author(s) 2016. This article is published with open access at Springerlink.com

\begin{abstract}
A new macroscopic description of capillary transport of liquid and gas in porous materials is presented within the framework of multiphase continuum mechanics. It is assumed that unsaturated porous material form a macroscopic continuum composed of three constituents: gas, mobile liquid and capillary liquid, while the skeleton is rigid with an isotropic pore space structure. The capillary liquid forms a thin layer on the internal contact surface with the skeleton, is immoveable and contains the whole capillary energy. The remaining part of the liquid, surrounded by the layer of the capillary liquid and menisci, forms the constituent called mobile liquid. Both liquids exchange mass, momentum and energy in the vicinity of menisci surfaces during their motion, which is described by an additional macroscopic velocity field. A macroscopic scalar quantity is introduced, parametrizing changes of saturations, which for quasi static and stationary processes is equal to the capillary pressure. This quantity extends the dimensionality of the description of mechanical processes taking place in unsaturated porous materials. For the three fluid constituents of the medium, balance equations of mass and linear momentum and evolution equations for saturations are formulated. The constitutive relations
\end{abstract}

M. Cieszko ( $\square)$

Institute of Mechanics and Applied Computer Science, Kazimierz Wielki University, Bydgoszcz, Chodkiewicza 30, 85-064 Bydgoszcz, Poland

e-mail: cieszko@ukw.edu.pl for quantities describing mechanical processes in such a medium are proposed based on the dissipation inequality of mechanical energy for the whole system. A new approach is proposed, similar to that used in rational thermodynamics, based on entropy inequality analysis and the Lagrange multipliers method.

Keywords Unsaturated porous materials . Macroscopic description - Capillary transport

\section{Introduction}

The description and analysis of liquid and gas transport in unsaturated porous materials is very important in a number of engineering fields. These include, for example, porosimetry [20, 21, 69, 70] and engineering of porous materials [1, 42], groundwater flow [57, 73], hydraulic engineering, oil and gas mining [3], geophysics [54, 56] and drying processes $[41,53]$. It enables a thorough understanding and prediction of the course of phenomena in such materials, and consequently also, for example, determination of precise material characteristics, effective control of transport processes, and production of porous materials with desired physical properties.

The course of phenomena in unsaturated porous materials is generally highly complex. It depends not only on the physical properties of the porous skeleton and the liquid and gas filling its pores, but is 
additionally complicated by the interaction between constituents of the system during their relative motion and deformation. The nature of this interaction is determined by the surface phenomena at phase interfaces, and in particular by the capillary interactions. These are determined by the nature of the interaction between the liquid and the skeleton (wettability/non-wettability of the skeleton) and are closely related to the microscopic structure of the skeleton pore space. Due to the very large internal surface area of the liquid filling the pores of a porous material, rarely found in liquids outside the pore space, there are significant differences between the properties and behaviour of the liquid in the two states. These factors cause that the modelling of liquid and gas transport in unsaturated porous materials, at both microscopic and macroscopic levels, is a very complex problem, requiring the development of new concepts and methods of description.

The non-standard nature of the problem is revealed even in the relatively simple problem of the macroscopic description of the process of quasi-static intrusion of a non-wetting liquid (e.g. mercury) into a sample of homogeneous porous material. The situation here is unusual for hydrostatics, where in spite of the homogeneous pressure distribution in the liquid, the distribution of its saturation in the medium is inhomogeneous. In this case, the non-standard approach to solution of this problem is to formulate balance equations and constitutive relations for processes taking place in the pore space-pressure continuum, [15]. A flow of such liquid, e.g. through a layer of porous material can be considered as some disturbance of the process of its quasi-static intrusion into layer that violate the balance of pressures at both sides of the layer. Such flow is similar to a flow of liquid through a tube with a changeable cross-section the diameter of which depends on local value of pressure. A description of such flow requires that the strongly coupled fields of pressure, velocity and diameter (saturation) be determined. In the case of flow through porous material, the problem becomes even more complex, since all fields have to be expressed in the language of continuum mechanics.

Another example of the complexity of the macroscopic description of liquid and gas transport in an unsaturated porous material is the kinematics of the motion of both constituents. An important factor determining the kinematics of the liquid and gas, apart from the hydrodynamic motion of the constituents in the regions of the pore space that they currently occupy, is the motion of the menisci causing the evolution of the occupied regions. This is particularly significant since the mechanism of the menisci motion is other than that of both fluids, and requires different constitutive description.

Equations describing the flow of liquid in unsaturated porous material were first proposed more than 80 years ago by Richards, [62]. These equations were formulated for the capillary potential of a liquid generalizing Darcy's law to flow in unsaturated porous materials. The assumption was made that both the capillary potential of the liquid and the coefficient of permeability appearing in the law are unique functions of the liquid content. A simplified description was obtained in which the experimentally determined parameters of the model are complex functions of the liquid content.

Another approach was proposed by Leverett [44], who introduced into the theory of multiphase flow, instead of the capillary potential of the liquid, the capillary pressure, as a constitutive quantity uniquely determined by the saturation with the liquid. This assumption became a basic constitutive relation in the theory discussed in many monographic works in the field [5, 17, 31, 48, 58, 64]. It is a very strong assumption, which has a number of important consequences. For example, from the assumption it follows directly that in equilibrium states of the medium, when the pressure distribution in the liquid and in the gas is homogeneous (neglecting the effect of the gravity), the distribution of the saturation of the medium with liquid is also homogeneous, irrespective of the history of the process and the shape and size of the sample in which it took place. Inversely, every inhomogeneity in the distribution of the liquid in the porous medium causes flow of liquid.

Even the sophisticated thermodynamic models of two-phase flow in porous materials, presented in [23, 27, 33, 34, 39], do not describe an inhomogeneous distribution of liquid in quasi-static processes. In these works the capillary pressure in equilibrium is defined as a constitutive quantity, which, besides the saturation with liquid, can also be a function of other parameters of the system state: mass density, temperature, and specific interphase surface area.

A proposal for the description of the flow of two liquids in a porous material without using the 
constitutive relation for capillary pressure is contained in [36]. A key element of this description is the division of the regions occupied by each of the liquids into percolating and nonpercolating subregions [37, 38]. The liquids in percolating regions remain in contact with the liquid outside the medium, and can flow hydrodynamically, while the liquids in the nonpercolating regions are trapped by the capillary forces. Mass exchange takes place between the liquids in the two subregions. It is assumed that the capillary forces are of the nature of potential body forces, and the forms of constitutive relations for the capillary potentials, the stress tensors in the trapped liquids and the rate of mass exchange between parts of both liquids are proposed. As a result, a multi-parametric description is obtained for the flow in a porous material, which in static states of the medium reduces to the form of a homogeneous distribution of percolating liquids and an inhomogeneous distribution of trapped liquids. This means, for example, that in the processes of quasi-static intrusion of a non-wetting liquid into a porous material, where part of the medium is empty, the whole of the liquid intruded is a trapped liquid. Therefore, this model cannot be used for the description of processes of this type.

Perhaps, the application of the second gradient theory of fluid displacement, proposed in the paper [47], makes such description possible. The second gradient theory is used in mechanics as a thermodynamic model of continuous media to study capillarity in fluids $[18,30,31]$ and other phenomena $[2,19,30$, $47,59-61,67]$ like damage [60] and plasticity [59] as well as in a micro-structural model for the dissipation phenomena in concrete [63].

Constructive criticism of deficiencies and inconsistencies of existing macroscale models and averaging based approaches to modelling of transport phenomena in porous medium systems is given in paper [24]. This paper and [50] are devoted to presentation of a framework of the thermodynamically constrained averaging theory (TCAT), which in the opinion of the authors is a universal methodology for generating consistent multiphase porous medium models. The TCAT is based upon rigorous averaging procedures of microscale conservation equations and thermodynamic relations, and is formulated in opposition to the macroscopic modelling approach. In this method, the obtained entropy inequality for the whole system and macroscopic balance equations for constituents are used for derivation of constitutive relations for the modelled system, based on the Lagrange multipliers method. Applications of this methodology are demonstrated in papers $[25-27,39,51]$ and summarised in [28].

The similar approach is presented in papers $[7,8]$ devoted to formulation of description of multiphase, multicomponent thermodynamics of swelling porous media which additionally incorporate quasi static effects of electrical fields.

In the opinion of the author of the present paper, both approaches to formulating macroscale models; based on averaging procedures and macroscopic modelling, are not in opposition to each other, but rather are complementary. Each of them has advantages and disadvantages, and none of them provides a recipe for the creation new concepts of macroscopic models.

The characteristic feature of methods based on averaging procedures is direct transfer of the image of modeled process from the microscopic to the macroscopic level. This provides direct correspondence of macroscopic quantities with quantities describing processes at the microscopic level. However, the obtained macroscopic image of the modeled process is rather a mixture of macroscopic and microscopic concepts, and derived equations have complex structure and contain many coefficients that must be determined experimentally.

Macroscopic models of processes in complex systems like multiphase porous materials cannot be a simple transfer of the image of these processes from the microscopic level. Building a macroscopic model requires a very careful study of the essence of the course of processes at the microscopic level and the proposition of how to reflect these processes in internal structure of the macroscopic model. A macroscopic model should give a new insight into the course of the modelled processes, and therefore it often requires new concepts and sometimes also new tools to be introduced for their description. Such an approach is characteristic for the method based on the framework of rational continuum mechanics and thermodynamics $[16,22,55,68]$. Due to the great role that such approach has played in development of single-phase continuum mechanics and thermodynamics, it is expected that this macroscopic modelling should play the similar role in development of 
description of transport processes in unsaturated porous materials.

Some example showing that this direction of investigations is possible and can be fruitful, is very complex problem of fluid motion in saturated porous materials with anisotropic pore space structure and the related problem of its parametric characterization. In papers [12-14] this problem was solved by application of the anisotropic metric space (Minkowski space) as a macroscopic model of anisotropic pore space of rigid permeable material in which fluid flow takes place. This made possible among other things, precise definition of the tortuosity parameter for anisotropic pore space and to show that the metric tensor of the anisotropic space defines the anisotropic properties of the tortuosity parameter. This tensor can be interpreted as the tortuosity tensor of the anisotropic pore space. Fluid motion in this space is described in papers [12, 13] by mass and momentum balance equations and constitutive relations for fluid stress tensor and its interaction force with the skeleton.

The approach presented in these papers is pure macroscopic, characteristic for rational continuum mechanics and thermodynamics, and the obtained results are inaccessible for the methods based on averaging procedures. However, these procedures are indispensable e.g. for relating the tortuosity tensor as a macroscopic quantity with the microscopic geometry of the pore space. This example shows the importance of macroscopic modeling for proper formulation of the consistent and comprehensive continuum theory.

It may be supposed that at the present level of development of continuum theory of multi-phase media, formulation of a general macroscopic theory even for mechanics of multi-phase transport in porous media must have an evolutionary character. Formulation of such description needs solution of many partial problems. This concerns not only macroscopic description of capillary transport in porous media but also its relation to the pore space structure that may be anisotropic and evolve during deformation of the skeleton. The problem becomes much more complicated when the skeleton has anisotropic mechanical properties or thermal and electrical phenomena are taken into account.

In the present work, a new macroscopic description is proposed for the capillary transport of liquid and gas in porous materials of the homogeneous and isotropic pore space structure. Theoretical considerations are conducted within the framework of the multiphase continuum mechanics and are based on three key model assumptions. This concern:

- division of liquid in the pore space into two macroscopic constituents called mobile liquid and capillary liquid,

- description of menisci motion by an additional macroscopic velocity field,

- parametrization of saturation changes by a macroscopic pressure-like quantity that for quasi static and stationary processes is equal to the capillary pressure.

The division of liquid into two continua is justified both kinematically and energetically. The capillary liquid forms a film on its contact surface with the skeleton. This liquid is immobile and contains the whole interfacial (capillary) energy of liquid in the pore space. The remaining part of the liquid surrounded by the layer of capillary liquid and menisci forms the mobile liquid. Both liquids exchange mass, linear momentum and energy in the vicinity of menisci surfaces and this occurs only during menisci motion in the pore space.

The additional velocity field describing menisci motion reflects the complexity of kinematics in unsaturated porous materials and enables the modeling of mechanisms of menisci motion in the pore space.

Parametrization of saturation extends the dimensionality of the description of processes taking place in an unsaturated porous material enabling, among others, the description of liquid distribution in quasi-static processes and in the stationary flow.

Balance equations of mass and linear momentum are formulated for fluid constituents of the unsaturated porous material, and constitutive relations are derived for quantities describing mechanical processes in such a medium. It is based on the analysis of the dissipation inequality of mechanical energy formulated for the whole system. A new approach is proposed, similar to that used in the rational thermodynamics, based on entropy inequality analysis and the Lagrange multipliers method. 


\section{Basic assumptions}

\subsection{Kinematic constituents of the medium}

Capillary phenomena in unsaturated porous materials are closely related to the existence of the enlarged internal surface layer of the liquid in the pore space. The physical properties of the liquid in this layer substantially differ from those of the liquid filling internal parts of the pore space (bulk liquid) and its energetic state and spatial configuration depends on the character of the physical interaction between the liquid and the skeleton material (wettable/nonwettable skeleton), on the geometry of the internal pore space structure and the course taken by the process in the medium (Fig. 1). For example, in the processes of intrusion of a nonwetting liquid (e.g. mercury) into a porous material, schematically shown in Fig. 1a and in Fig. 1b, the work performed by the external forces is transformed into internal energy concentrated in the enlarged liquid film on its contact surface with the skeleton. A similar situation occurs when wetting liquid is extruded from porous material by gas (Fig. 1d). In this case, the internal surface layer of the liquid is present also in the region occupied by gas. There, it takes the form of double layer. During inflow of wetting liquid into dry porous material (Fig. 1c), a single liquid layer is formed on the surface of pores, the work is performed by the liquid on the extruded gas, and part of the liquid internal energy is changed into wetting heat.

From a kinematic point of view, the whole surface layer of the liquid in the pore space can be divided into two parts: the menisci surface layer and the adjacent surface layer. The menisci surface layer is formed of those parts of the liquid surface layer that are in contact with gas and can move together with the liquid and gas along the pores. The adjacent surface layer is the remaining part of the liquid surface layer. This surface is in direct contact with the skeleton and its area is substantially greater than that of the menisci surface. In regions occupied by the gas, the adjacent surface layer may form a double layer. It will be assumed that this surface layer is immobile at the skeleton pore surface and contains the whole interfacial energy of the liquid filling the pores of a porous material.

Because of the immobility of the adjacent surface layer, the internal energy of this layer cannot be treated as the internal energy of the whole liquid in the pore space. The internal energy of each constituent is transported with that constituent. This means that the adjacent surface layer should be regarded as a separate constituent of the medium. Such separation is justified both kinematically and energetically, and plays a very important role in the macroscopic model. It reflects the essence of the course of processes at the microscopic level in the structure of the macroscopic model. However, at the microscopic level, such division has an imaginary character, since there is no explicit physical surface separating both liquids. Nevertheless, to provide the uniqueness of the liquid division we assume that such separating surface exists and can be uniquely definite.

In view of direct connection of the liquid contained in the adjacent surface layer with capillary effects in unsaturated porous medium, it will be called a capillary liquid and its internal energy will be called capillary energy. This nomenclature corresponds to that used in literature (e.g. [9, 44, 62, 65]).

The remaining part of the liquid surrounded by the layer of capillary liquid and menisci will be called mobile liquid, as it can move in the pore space. In thermodynamic equilibrium the capillary liquid does not exchange mass and energy through the contact surface with the mobile liquid. Such exchange exists, however, in the vicinity of the menisci surfaces during their motion in the pore space.

Taking into account the above microscopic conditions of mass and energy transport in the unsaturated porous materials, for macroscopic description of capillary transport in such media we apply a treeconstituent continuum model composed of the capillary liquid, mobile liquid and gas. The skeleton is assumed to be rigid and immobile, with an isotropic pore space structure. Theoretical considerations will be conducted within the framework of multiphase continuum mechanics. This means that all quantities used in the paper are macroscopic, defined on the representative elementary volume.

\subsection{Parameterisation of menisci motion}

The menisci surface separating the mobile liquid and gas plays a very important role in the course of capillary processes in unsaturated porous materials. In the case when the capillary liquid is incompressible, current positions of menisci uniquely determine the 
Fig. 1 Schematic illustration of non-wetting liquid intrusion into a sample of porous material (a) and of three typical compositions of constituents in the medium: b nonwetting liquid intrusion, c wetting liquid inflow, d wetting liquid extrusion (a)
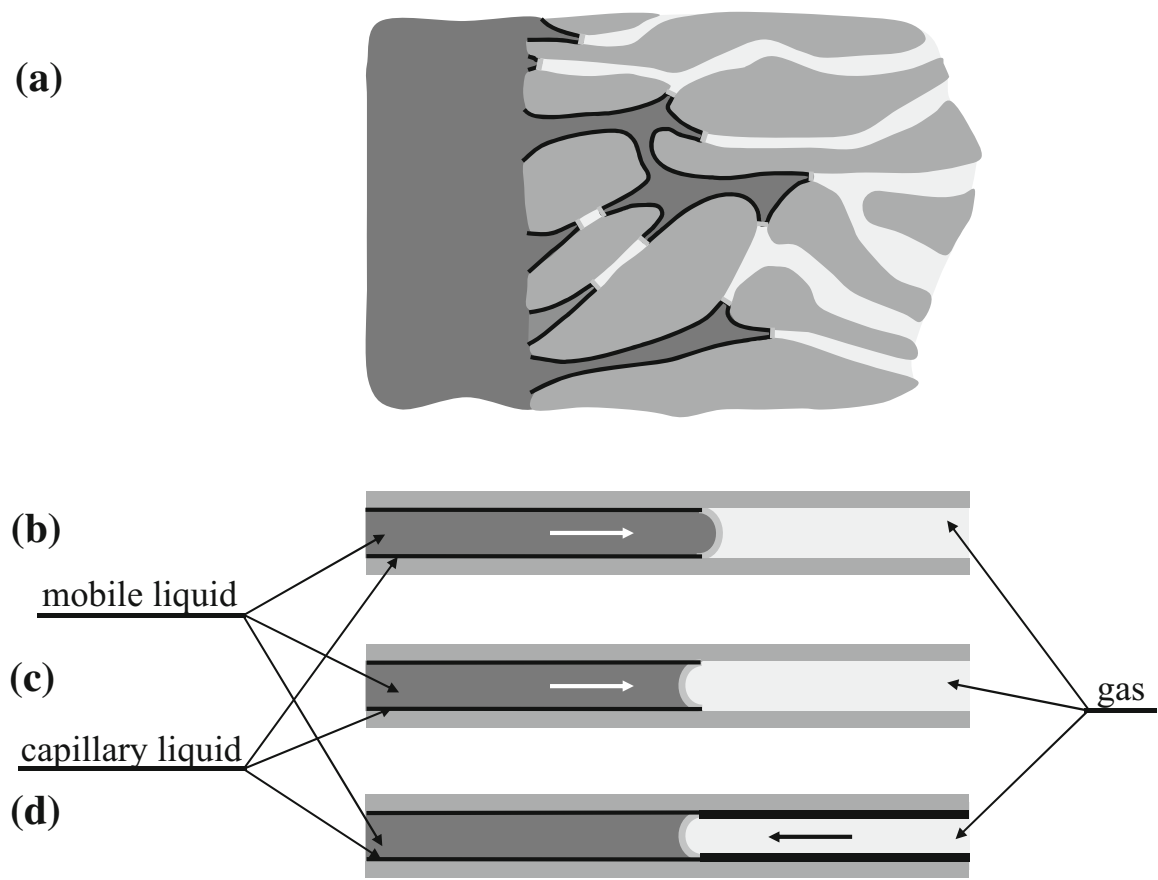

areas occupied by the fluid constituents of the medium in the pore space. The menisci surfaces can move during processes which occur in the medium, causing mass exchange between the mobile and the capillary liquid. The surfaces of menisci are places where such mass exchange occurs, and their motion is a necessary condition for this exchange.

Taking into account that in quasi-static processes the menisci motion is caused by changes in the static capillary pressure $\hat{p}_{c}$, defined as the difference of the pressure $p_{m}$ in the (mobile) liquid and pressure $p_{g}$ in the gas,

$\widehat{p}_{c}=p_{m}-p_{g}$,

it is assumed that in the general case of no stationary processes, a macroscopic scalar quantity $r$ exists which determines the motion of menisci. This quantity may be a function of spatial coordinate $\mathbf{x}$ and time $t$, $r=r(\mathbf{x}, t)$.

Changes in the quantity $r$ result in menisci motion, and fixing the quantity value at a new level stops the menisci motion in a new configuration. This macroscopic quantity can be interpreted as a pressure that in quasi-static and stationary processes is equal to the capillary pressure $\left(r \equiv \widehat{p}_{c}\right)$. It may be supposed that also in processes with a slow evolution of saturations, the quantity $r$ can be approximated by the capillary pressure,

$r=r(\mathbf{x}, t) \cong \hat{p}_{c}=p_{m}-p_{g}$.

Such an interpretation makes possible description of a very large class of quasi static and stationary problems in unsaturated porous materials, and can also be used for description of non-stationery problems the dynamics of which is not too high.

It should be underlined that the capillary pressure $\hat{p}_{c}$ defined by the formula (1) does not determine the stress state in the capillary liquid as a constituent of the proposed model of the medium. The capillary pressure and the pressure in the capillary liquid are two different quantities.

\section{Kinematics and equations for mass balances and for the evolution of saturations}

The macroscopic description of the motion of constituents of an unsaturated porous medium is made within the framework of multi-phase continuum mechanics. It is assumed that from the macroscopic point of view each constituent forms a material 
continuum, generally understood as a medium with continuous spatial distributions of mass and mass flux. This approach enables one to define the basic notions of the kinematics of these constituents within the terms of spatial description of the medium, without the need to use terms related to a macroscopic particle of the fluid, whose material identity during its motion in the pore space is not certain.

\subsection{Partial and phase densities of the constituents}

It is assumed that a representative elementary volume $d V$ exists of the space occupied by the unsaturated porous medium in which all of the macroscopic quantities describing the kinematics of the constituents: the mobile and capillary liquid and the gas, have appropriate stochastic representations. Then, the masses $d m_{m}, d m_{c}, d m_{g}$ of these constituents contained in the volume element $d V$ can be represented in the form:

$d m_{m}=\bar{\rho}_{m} d V, \quad d m_{c}=\bar{\rho}_{c} d V, \quad d m_{g}=\bar{\rho}_{g} d V$,

where $\bar{\rho}_{m}, \bar{\rho}_{c}, \bar{\rho}_{g}$ are partial densities of the constituents. They are linked to the phase densities $\rho_{m}, \rho_{c}, \rho_{g}$ by the relations

$\bar{\rho}_{m}=f_{v} s_{m} \rho_{m}, \quad \bar{\rho}_{c}=f_{v} s_{c} \rho_{c}, \quad \bar{\rho}_{g}=f_{v} s_{g} \rho_{g}$,

where $f_{v}$ is the volume porosity of the medium, and $s_{m}, s_{c}, s_{g}$ respectively denote saturations with the mobile and capillary liquid and the gas. The saturation is defined as the ratio of the volume occupied by the given constituent in a representative sample of an unsaturated medium to the pore volume in that sample. The saturations are related by the identity

$s_{m}+s_{c}+s_{g}=1$.

In accordance with the remarks made in the previous section, menisci motion and mass exchange between the mobile and capillary liquid do not occur in an unsaturated porous material when the parameter $r$ does not change. In such a case, changes in the saturation parameters of the medium with both liquid and gas will be caused only by density changes of the medium constituents. If we assume that the capillary liquid is incompressible, then the saturation with the capillary liquid will not change when there is no menisci motion. As a result, saturation with the mobile liquid and the gas will also be constant since they are in contact only through the surfaces of menisci. Under this assumption, the current positions of menisci uniquely determine the areas occupied by the constituents of the medium in the pore space. This also means that, in general, saturation parameters should additionally be functions of the quantity $r$, and that they cannot be directly time-dependent, because it is not possible for them to change when the value of the quantity $r$ is fixed. We assume

$s_{m}=s_{m}(\mathbf{x}, r(\mathbf{x}, t)), \quad s_{c}=s_{c}(\mathbf{x}, r(\mathbf{x}, t))$,
$\quad s_{g}=s_{g}(\mathbf{x}, r(\mathbf{x}, t))$.

However, these functions have to depend on the spatial coordinate $\mathbf{x}$ explicitly, because otherwise the homogeneity of the quantity $r$, e.g. in quasi-static processes, would result in a homogeneous distribution of fluid saturations in the porous material. In this case, menisci motion is the only possibility for changing the fluid saturations in the medium.

The functions (6) will be explicitly time-dependent only when the capillary liquid is compressible. In general, the saturations in (6) will be given by the expressions

$s_{m}=s_{m}(\mathbf{x}, t, r(\mathbf{x}, t)), \quad s_{c}=s_{c}(\mathbf{x}, t, r(\mathbf{x}, t))$,
$\quad s_{g}=s_{g}(\mathbf{x}, t, r(\mathbf{x}, t))$.

These saturations are explicit functions of the quantity $r$. Their functional dependence on this quantity has to be determined from differential equations describing the proposed model. The form of this dependence extends the dimensionality of processes taking place in unsaturated porous materials. It is explicitly visible already in description of a relatively simple problem of quasi-static intrusion of non-wetting liquid into a porous body, considered in paper [15] as a process taking place in the pore spacepressure continuum. This additional dimensionality determines the complexity of processes of capillary transport in unsaturated porous materials. Therefore, equations describing these processes have to be strongly coupled and nonlinear.

\subsection{Mass fluxes of the constituents}

To define fluxes of mass transport and velocity fields for each kinematic constituent of the unsaturated porous medium, we consider an infinitesimal 
representative element $d \mathbf{S}$ of the control surface $\partial D$ surrounding a region $D$ of the space,

$d \mathbf{S}=\mathbf{n} d S$,

where $d S$ is the area of the element, and $\mathbf{n}$ is a unit vector perpendicular to the surface element, externally oriented with respect to the region $D$.

On account of the immobility of the capillary liquid, its mass flux $d \Phi_{c}$ flowing through the surface element $d \mathbf{S}$ is equal to zero,

$d \Phi_{c}=0$.

The other two fluid constituents of the unsaturated porous material - the mobile liquid and the gas - can move in the medium. Therefore, their mass fluxes $d \Phi_{m}$ and $d \Phi_{g}$ flowing through the surface element $d \mathbf{S}$ take non-zero values. They are linked to the vectors of partial mass fluxes

$\overline{\mathbf{q}}_{m}=f_{v} s_{m} \mathbf{q}_{m}, \quad \overline{\mathbf{q}}_{g}=f_{v} s_{g} \mathbf{q}_{g}$,

by the relations

$d \Phi_{m}=\overline{\mathbf{q}}_{m} \cdot d \mathbf{S}, \quad d \Phi_{g}=\overline{\mathbf{q}}_{g} \cdot d \mathbf{S}$,

where $\mathbf{q}_{m}$ and $\mathbf{q}_{g}$ are the phase mass fluxes of the constituents, and the dot denotes the scalar product of vectors.

Menisci motion in the unsaturated porous materials plays a very important role in the kinematics of constituents of such media, strongly influencing motion of the mobile liquid and gas. Their motion defines changes of regions occupied by the constituents of the medium, and its mechanism is completely different from the hydrodynamic mechanism of fluid motion in regions they currently occupy. Therefore, both motions have to be considered as independent and should be defined by different physical laws. It may happen that, in spite of motion of the fluids, the menisci do not move. However, every change of menisci position is associated with motion of some parts of the mobile liquid and the gas. This means that the local kinematics of both mobile constituents (the mobile liquid and the gas) is determined by two factors: menisci motion and the free motion of each constituent in the regions they currently occupy. It is assumed, therefore, that the partial mass fluxes of the mobile liquid and the gas have two components. The first component is formed by the mass fluxes $\overline{\mathbf{q}}_{m}^{c}$ and $\overline{\mathbf{q}}_{g}^{c}$ associated with the motion of menisci in regions of inhomogeneous distributions of saturations with both fluids. These fluxes will be used in defining the additional velocity field describing motion of the menisci in an unsaturated porous material. Independently of this, there is also the volumetric motion of menisci in the pore space that should be described by the volume source in the evolution equations for saturations.

The second component of the partial mass fluxes of the mobile liquid and the gas is formed by the mass fluxes $\overline{\mathbf{q}}_{m}^{f}$ and $\overline{\mathbf{q}}_{g}^{f}$ related to the free motion of both constituents in regions they currently occupy.

The partial mass fluxes of the mobile liquid and the gas can be represented in the form

$\overline{\mathbf{q}}_{m}=\overline{\mathbf{q}}_{m}^{c}+\overline{\mathbf{q}}_{m}^{f}, \quad \overline{\mathbf{q}}_{g}=\overline{\mathbf{q}}_{g}^{c}+\overline{\mathbf{q}}_{g}^{f}$.

It is assumed moreover that the partial fluxes $\overline{\mathbf{q}}_{m}^{c}$ and $\overline{\mathbf{q}}_{g}^{c}, \overline{\mathbf{q}}_{m}^{f}$ and $\overline{\mathbf{q}}_{g}^{f}$ are linked to phase fluxes $\mathbf{q}_{m}^{c}$ and $\mathbf{q}_{g}^{c}, \mathbf{q}_{m}^{f}$ and $\mathbf{q}_{g}^{f}$ by the relations

$\overline{\mathbf{q}}_{m}^{c}=f_{v} s_{m} \mathbf{q}_{m}^{c}, \quad \overline{\mathbf{q}}_{m}^{f}=f_{v} s_{m} \mathbf{q}_{m}^{f}$,

$\overline{\mathbf{q}}_{g}^{c}=f_{v} s_{g} \mathbf{q}_{g}^{c}, \quad \overline{\mathbf{q}}_{g}^{f}=f_{v} s_{g} \mathbf{q}_{g}^{f}$.

Therefore, the total phase mass fluxes $\mathbf{q}_{m}$ and $\mathbf{q}_{g}$ of the mobile liquid and the gas are

$\mathbf{q}_{m}=\mathbf{q}_{m}^{c}+\mathbf{q}_{m}^{f}, \quad \mathbf{q}_{g}=\mathbf{q}_{g}^{c}+\mathbf{q}_{g}^{f}$.

Taking into account that fluxes $\mathbf{q}_{m}^{c}$ and $\mathbf{q}_{g}^{c}$ of the mobile liquid and the gas associated with the menisci motion should take zero value when the menisci do not move, these mass fluxes have to be functions of local changes in the quantity $r$ which defines their motion. Therefore, we take representations of these fluxes in the form

$\mathbf{q}_{m}^{c}=\mathbf{q}_{m}^{r} \frac{\partial r}{\partial t}, \quad \mathbf{q}_{g}^{c}=\mathbf{q}_{g}^{r} \frac{\partial r}{\partial t}$,

where $\mathbf{q}_{m}^{r}$ and $\mathbf{q}_{g}^{r}$ are the mass fluxes of the mobile liquid and the gas, respectively, associated with the menisci motion in $r$-space, i.e. fluxes referred to the unit value of the parameter $r$. The $r$-space is the analogue of the time-space.

The representations of mass fluxes (16) use local derivatives of the parameter $r$, because in the stationary processes menisci motion does not occur in the medium and these fluxes should take zero value. 
3.3 Velocity fields of the constituents and menisci

We use the phase fluxes $\mathbf{q}_{m}^{c}$ and $\mathbf{q}_{g}^{c}$ describing the mass transport of parts of the mobile liquid and the gas associated with the menisci motion to define the velocity field $\mathbf{v}^{c}(\mathbf{x}, r)$ of their motion. We require the velocities of both fluid parts to be equal to the velocity of menisci motion. The definition of this velocity takes the form

$\mathbf{v}^{c}(\mathbf{x}, r) \equiv \frac{\mathbf{q}_{m}^{c}}{\rho_{m}} \equiv \frac{\mathbf{q}_{g}^{c}}{\rho_{g}}$.

Due to representations (16) we obtain

$$
\mathbf{v}^{c}(\mathbf{x}, r) \equiv \mathbf{v}_{r}(\mathbf{x}, r) \frac{\partial r}{\partial t},
$$

where the quantity

$\mathbf{v}_{r}(\mathbf{x}, r) \equiv \frac{\mathbf{q}_{m}^{r}}{\rho_{m}} \equiv \frac{\mathbf{q}_{g}^{r}}{\rho_{g}}$

may be interpreted as the "velocity" of menisci motion in the $r$-space. This quantity characterises the rate of the change in menisci position in the space as the value of the parameter $r$ changes.

Similarly, using the phase fluxes $\mathbf{q}_{m}^{f}$ and $\mathbf{q}_{g}^{f}$, velocity fields for the motion of free parts of the mobile liquid and the gas can be defined:

$\mathbf{v}_{m}^{f}(\mathbf{x}, t)=\frac{\mathbf{q}_{m}^{f}}{\rho_{m}}, \quad \mathbf{v}_{g}^{f}(\mathbf{x}, t)=\frac{\mathbf{q}_{g}^{f}}{\rho_{g}}$,

Considering expressions (13)-(19), the mass fluxes (12) for the mobile liquid and the gas can be represented in the form

$\overline{\mathbf{q}}_{m}=\bar{\rho}_{m}\left(\mathbf{v}_{r} \frac{\partial r}{\partial t}+\mathbf{v}_{m}^{f}\right)$,

$\overline{\mathbf{q}}_{g}=\bar{\rho}_{g}\left(\mathbf{v}_{r} \frac{\partial r}{\partial t}+\mathbf{v}_{g}^{f}\right)$,

where the partial densities $\bar{\rho}_{m}$ and $\bar{\rho}_{g}$ are given by (4).

From (21) and (22) it results that the velocity fields $\mathbf{v}_{m}^{f}$ and $\mathbf{v}_{g}^{f}$ describe the motion of the mobile liquid and the gas at fixed distributions of medium saturation with both constituents. Expressions (21) and (22) make it possible to define the resultant velocity fields for the mobile liquid and the gas. We have

$$
\mathbf{v}_{m}=\frac{\mathbf{q}_{m}}{\rho_{m}}=\mathbf{v}_{r} \frac{\partial r}{\partial t}+\mathbf{v}_{m}^{f}, \quad \mathbf{v}_{g}=\frac{\mathbf{q}_{g}}{\rho_{g}}=\mathbf{v}_{r} \frac{\partial r}{\partial t}+\mathbf{v}_{g}^{f} .
$$

Introduction of the additional velocity field that describes motion of the menisci in the pore space is also important for the constitutive modeling of liquid and gas transport in unsaturated porous materials. This quantity enables constitutive modeling of the mechanism of menisci motion in the pore space.

\subsection{Mass balance equations for the constituents}

On account of the immobility of the capillary liquid and mass exchange with the mobile liquid, its local mass balance equation can be presented in the form

$\frac{\partial \bar{\rho}_{c}}{\partial t}=\bar{\psi}$

where $\bar{\psi}$ represents a volume source of mass of the capillary liquid. It defines the mass of the capillary liquid exchanged with the mobile liquid in a unit of time and in a volume unit of space occupied by the medium. It is related to the quantity $\psi$ referred to a unit volume of the capillary liquid by

$\bar{\psi}=f_{v} s_{c} \psi$.

Considering the representations (21) and (22) for the mobile liquid and gas fluxes, and in view of the mass exchange between the mobile and the capillary liquid, the local mass balance equations for the mobile liquid and the gas can be written as follows

$\frac{\partial \bar{\rho}_{m}}{\partial t}+\operatorname{div}\left(\bar{\rho}_{m} \mathbf{v}_{m}\right)=-\bar{\psi}$,

$\frac{\partial \bar{\rho}_{g}}{\partial t}+\operatorname{div}\left(\bar{\rho}_{g} \mathbf{v}_{g}\right)=0$

where the resultant velocity fields $\mathbf{v}_{m}$ and $\mathbf{v}_{g}$ of both constituents are given by (23).

\subsection{Evolution equations for saturations}

Menisci motion in the pore space of unsaturated porous materials is described by the velocity field $\mathbf{v}^{c}(\mathbf{x}, r)$ defined by the identity (17). This quantity determines the changes of regions occupied by particular constituents, and therefore, have to be directly related with changes of the parameters of 
pore saturation with the constituents. These relations should have a pure geometrical character reflecting the balance of volume changes of regions occupied by the constituents in the pore space. Taking into account that menisci motion in the pore space may also have a volumetric character, such balances will have the form of mass balance equations with sources, formulated for media of unit densities.

The balance equations of changes of volume occupied by the mobile and the capillary liquids and by the gas can be written in the form

$\frac{\partial^{\mathrm{T}} s_{m}}{\partial t}+\frac{\partial^{\mathrm{T}} s_{c}}{\partial t}+\operatorname{div}\left(s_{m} \frac{\partial r}{\partial t} \mathbf{v}_{r}\right)=\psi_{m c}^{\mathrm{v}}$

$\frac{\partial^{\mathrm{T}} s_{g}}{\partial t}+\operatorname{div}\left(s_{g} \frac{\partial r}{\partial t} \mathbf{v}_{r}\right)=\psi_{g}^{\mathrm{v}}$

where

$\frac{\partial^{T}}{\partial t}=\frac{\partial}{\partial t}+\frac{\partial}{\partial r} \frac{\partial r}{\partial t}$

and $\psi_{m c}^{\mathrm{v}}$ as well as $\psi_{g}^{\mathrm{v}}$ represent source components of the volume changes. These components have to be dependent quantities because the whole region occupied by three fluid constituents (the pore space) does not change.

Due to the identity (5), from Eqs. (28) and (29) we obtain

$\operatorname{div}\left(\left(1-s_{c}\right) \frac{\partial r}{\partial t} \mathbf{v}_{r}\right)=\psi_{m c}^{\mathrm{v}}+\psi_{g}^{\mathrm{v}}$.

Equation (30) defines the relation between quantities $\psi_{m c}^{\mathrm{v}}$ and $\psi_{g}^{\mathrm{v}}$. This means that Eqs. (28) and (29) are equivalent and only one of them describes the evolution of the saturation in unsaturated porous materials. The source component occurring in one of these equations requires the definition by the constitutive relation.

\section{Momentum balance equations for the constituents}

The assumed three-constituent model of the kinematics of the unsaturated porous medium requires the formulation of three momentum balance equations for the capillary and mobile liquids and the gas. Since the skeleton is non-deformable and immoveable, its momentum balance equation will be omitted, and it will be treated as isotropic space with which the fluid constituents of the medium can interact.

Surface interactions between constituents of the medium at the microscopic level depend on the surface properties of the liquid in contact with the skeleton and on the course taken by the process in the medium (Fig. 1). Taking into account that a layer of the capillary liquid separates the mobile liquid and the skeleton, they cannot interact directly. Surface interaction, however, may occur between the capillary liquid and the other two constituents. For wetting liquids, the regions occupied by the gas are places where surface interactions between the gas and the capillary liquid or the skeleton occur (Fig. 1c, d). For non-wetting liquids, such interactions always take place between the gas and the skeleton (Fig. 1a, b).

A particular role in interactions between constituents of the unsaturated porous material is performed by menisci. This is where all three constituents of the medium and the skeleton interact and the only place at which the gas interacts directly with the mobile liquid. Both constituents, in turn, interact through the menisci with the capillary liquid and the skeleton. On account of the lack of relative motion of the mobile liquid and gas in the vicinity of menisci, their interactions are not dissipative.

Interactions of the constituents of the medium occurring at the microscopic level will be modelled at the macroscopic level as volume (or mass) interactions.

\subsection{Momentum balance equation for the capillary liquid}

Taking into account that, in general, the capillary liquid may be in direct contact with the skeleton, the mobile liquid and the gas (Fig. 1d), it will be subjected to forces by all of the three constituents of the medium. Since the capillary liquid exchanges mass with the mobile liquid, the local form of its momentum balance equation can be presented as follows:

$\operatorname{div}\left(\overline{\mathbf{T}}_{c}\right)+\overline{\boldsymbol{\pi}}_{c s}+\overline{\boldsymbol{\pi}}_{c m}+\overline{\boldsymbol{\pi}}_{c g}+\bar{\rho}_{c} \mathbf{g}+\bar{\psi} \mathbf{w}=\mathbf{0}$,

where $\overline{\mathbf{T}}_{c}$ is the Cauchy tensor of partial stresses in the capillary liquid, related to the phase stress tensor $\mathbf{T}_{c}$ by $\overline{\mathrm{T}}_{c}=f_{v} s_{c} \mathrm{~T}_{c}$. Vectors $\overline{\boldsymbol{\pi}}_{c s}, \overline{\boldsymbol{\pi}}_{c m}, \overline{\boldsymbol{\pi}}_{c g}$ are the volume densities of interaction forces of the skeleton, the 
mobile liquid and the gas, respectively, with the capillary liquid. They are related to the mass densities $\pi_{c s}, \pi_{c m}, \pi_{c g}$ of interaction forces by

$\bar{\pi}_{c s}=\bar{\rho}_{c} \pi_{c s}, \quad \bar{\pi}_{c m}=\bar{\rho}_{c} \pi_{c m}, \quad \bar{\pi}_{c g}=\bar{\rho}_{c} \pi_{c g}$.

In processes of saturation of dry porous materials (Fig. 1a-c), there is no surface contact between the capillary liquid and gas. Then, the force $\bar{\pi}_{c g}$ will not be present in Eq. (31).

The quantity $\bar{\rho}_{c} \mathbf{g}$ in Eq. (31) represents volume density of the gravitational force acting on the constituent, and $\mathbf{g}$ is the gravitational acceleration vector. The term $\bar{\psi} \mathbf{w}$ denotes the momentum exchange associated with the mass exchange between the mobile and capillary liquids in a unit volume of the medium and vector $\mathbf{w}$ is the velocity of the exchanged mass.

\subsection{Momentum balance equation for the mobile liquid}

Considering that the mobile liquid is not in direct contact with the skeleton, it can interact only with the capillary liquid and the gas. However, its interaction with the gas cannot be described by volume forces, because both fluids are in contact through the menisci surfaces only. In multi-phase continuum mechanics, such interactions are included into the description during formulation of the stress constitutive relations. Therefore, the only volume interaction force exerted on the mobile liquid is the interaction force $\overline{\boldsymbol{\pi}}_{m c}$ with the capillary liquid:

$\bar{\pi}_{m c}=-\bar{\pi}_{c m}$.

This force contains at least two different components: the viscous interaction force due to the relative motion of the constituents, and the interaction force resulting from the inhomogeneous distribution of liquid in the medium.

Owing to the momentum exchange associated with the mass exchange between the mobile and capillary liquids described by the term $-\bar{\psi} \mathbf{w}$ and the mass balance Eq. (26) for the mobile liquid, the local form of the momentum balance equation for this liquid is

$\bar{\rho}_{m} \frac{D_{m} \mathbf{v}_{m}}{D t}=\operatorname{div}\left(\overline{\mathrm{T}}_{m}\right)+\overline{\boldsymbol{\pi}}_{m c}+\bar{\rho}_{m} \mathbf{g}-\bar{\psi}\left(\mathbf{w}-\mathbf{v}_{m}\right)$,
$D_{m}() / D t=\partial() / \partial t+\mathbf{v}_{m} \cdot \operatorname{grad}()$,

where $\mathbf{v}_{m}$ is the resultant velocity of the mobile liquid, given by expression $(23)_{1}$. The quantity $\overline{\mathrm{T}}_{m}$ is the Cauchy tensor of partial stresses in the mobile liquid, related to the phase stress tensor $\mathrm{T}_{m}$ by $\overline{\mathrm{T}}_{m}=f_{v} s_{m} \mathrm{~T}_{m}$. The volume density $\bar{\pi}_{m c}$ of the interaction force is related to its mass density $\pi_{m c}$ by $\bar{\pi}_{m c}=\bar{\rho}_{m} \pi_{m c}$. The last term in Eq. (33) represents the recoil force due to mass exchange between the mobile and capillary liquids.

\subsection{Momentum balance equation for the gas}

Taking into account that interaction forces between the gas and the mobile liquid through the menisci surfaces will be included in the description during the formulation of constitutive relations, the only volume interaction forces which should be considered in the momentum balance equation for the gas are the gravitational force $\bar{\rho}_{g} \mathbf{g}$ acting on the gas and its interaction force $\bar{\pi}_{g c}=-\bar{\pi}_{c g}\left(\bar{\pi}_{g s}=-\bar{\pi}_{s g}\right)$ with the capillary liquid (skeleton). Considering the mass balance Eq. (27) for the gas, the local form for its momentum balance equation can be written as follows:

$\bar{\rho}_{g} \frac{D_{g} \mathbf{v}_{g}}{D t}=\operatorname{div}\left(\overline{\mathrm{T}}_{g}\right)+\bar{\pi}_{g c}+\bar{\rho}_{g} \mathbf{g}$,

$D_{g}() / D t=\partial() / \partial t+\mathbf{v}_{g} \cdot \operatorname{grad}()$,

where $\mathbf{v}_{g}$ is the resultant velocity field of the gas, given by expression $(23)_{2}$, and $\overline{\mathrm{T}}_{g}=f_{v} s_{g} \mathrm{~T}_{g}$ is the Cauchy tensor of partial stresses in the gas and $\mathrm{T}_{g}$ is its phase stress tensor.

The volume density $\bar{\pi}_{g c}$ of the interaction force is related to its mass density $\pi_{g c}$ by

$\bar{\pi}_{g c}=\bar{\rho}_{g} \pi_{g c}$.

This term of Eq. (34) should be replaced by the volume interaction force $\bar{\pi}_{g s}$ for processes in which the gas is in direct contact with the skeleton (Fig. 1a-c).

Mass balance Eqs. (24), (26) and (27), evolution Eq. (28) for saturation, linear momentum balance Eqs. (33) and (34), and the identity (5) form a system of eleven scalar equations containing independent and constitutive quantities. The independent quantities in 
these equations are represented by densities, saturations and velocities of the constituents:

$\rho_{m}, \rho_{c}, \rho_{g}, s_{m}, s_{c}, s_{g}, \mathbf{v}_{m}, \mathbf{v}_{g}$,

and the remaining unknown fields are constitutive quantities:

$r, \psi, \psi_{m c}^{\mathrm{v}}, \mathbf{v}_{r}, \mathbf{w}, \overline{\boldsymbol{\pi}}_{c m}, \overline{\boldsymbol{\pi}}_{c g}, \overline{\boldsymbol{\pi}}_{c s}, \overline{\mathrm{T}}_{m}, \overline{\mathrm{T}}_{c}, \overline{\mathrm{T}}_{g}$.

The constitutive quantities describe the physical properties of the constituents of the medium and their interactions, and each of them has to be defined by an individual constitutive relation. The exception is the volume density $\overline{\boldsymbol{\pi}}_{c s}$ of the interaction force between the capillary liquid and the skeleton that, due to the immobility of both constituents, should be defined by the linear momentum balance Eq. (31) for the capillary liquid.

The independent quantities (36) form a set of twelve scalar fields and should be defined by equations of the balance type. Therefore, the number of such equations in the mathematical model has to be equal to the number of independent scalar fields to provide the system of equations in the model to be closed. The luck of equality between the number of equations of the balance type in the proposed model and the number of independent scalar fields gives rise to supposition that one of the quantities in the set (36) is not independent. It will be shown in section six that saturations with the mobile and the capillary liquids have to be related by constitutive equation.

To formulate the constitutive relations for isothermal processes of liquid and gas transport in unsaturated porous materials, we apply dissipation inequality of the mechanical energy formulated for the whole system and the method of Lagrange multipliers.

\section{Energy balance equation for the whole system}

Using the previously defined quantities describing the state of particular constituents of the unsaturated porous medium, their mass and momentum balance equations, the internal energy balance equation for the whole system takes the form

$$
\begin{aligned}
& \bar{\rho}_{s} \frac{\partial u_{s}}{\partial t}+\bar{\rho}_{c} \frac{\partial u_{c}}{\partial t}+\bar{\rho}_{m} \frac{D_{m} u_{m}}{D t}+\bar{\rho}_{g} \frac{D_{g} u_{g}}{D t} \\
& \quad-\operatorname{tr}\left(\mathbf{D}_{m} \overline{\mathrm{T}}_{m}\right)-\operatorname{tr}\left(\mathbf{D}_{g} \overline{\mathrm{T}}_{g}\right) \\
& \quad+\overline{\boldsymbol{\pi}}_{m c} \cdot \mathbf{v}_{m}+\bar{\pi}_{g c} \cdot \mathbf{v}_{g}-\bar{\psi}\left(u_{m}-u_{c}+\mathbf{v}_{m}\right. \\
& \left.\quad \cdot\left(\mathbf{w}-\mathbf{v}_{m} / 2\right)\right)=0
\end{aligned}
$$

where $u_{s}, u_{c}, u_{m}$ and $u_{g}$ represent the specific internal energies of the skeleton, capillary liquid, mobile liquid and the gas, respectively, and $\bar{\rho}_{s}$ is the partial mass density of the skeleton linked to its phase density $\rho_{s}$ by the relation $\bar{\rho}_{s}=\left(1-f_{v}\right) \rho_{s}$. Quantity

$\mathbf{D}_{\alpha}=\frac{1}{2}\left(\nabla \otimes \mathbf{v}_{\alpha}+\mathbf{v}_{\alpha} \otimes \nabla\right)$

is the strain rate tensor of constituent $\alpha(\alpha=m, g)$ and $\otimes$ denotes the tensor product of vectors.

The specific internal energy of each constituent of the unsaturated porous medium can be presented as a sum of the Helmholtz free energy density $u_{\alpha}^{F}$ and thermal energy density that in thermodynamic equilibrium is equal to the product of the absolute temperature $T$ and specific entropy $\eta_{\alpha}$ (see e.g. in $[10,40]$. We have

$u_{\alpha}=u_{\alpha}^{F}+T \eta_{\alpha} \quad \alpha=s, c, m, g$.

Taking into account that the interfacial energy of the liquid filling porous material has been separated as an internal energy of the capillary liquid, the Helmholtz free energy of each constituent of the medium can be identified with the mechanical energy, linked with the deformation of the constituent. Therefore, the Helmholtz free energy density of the skeleton is assumed to be equal to zero $\left(u_{s}^{F}=0\right)$.

Considering representations (39) and omitting temporal and spatial changes of the temperature $T$, Eq. (38) can be presented in the form of balance equation for the strain energy of all fluid constituents in the unsaturated porous medium

$$
\begin{aligned}
& \bar{\rho}_{c} \frac{\partial u_{c}^{F}}{\partial t}+\bar{\rho}_{m} \frac{D_{m} u_{m}^{F}}{D t}+\bar{\rho}_{g} \frac{D_{g} u_{g}^{F}}{D t}-\operatorname{tr}\left(\mathbf{D}_{m} \overline{\mathbf{T}}_{m}\right)-\operatorname{tr}\left(\mathbf{D}_{g} \overline{\mathrm{T}}_{g}\right) \\
& \quad+\overline{\boldsymbol{\pi}}_{m c} \cdot \mathbf{v}_{m}+\overline{\boldsymbol{\pi}}_{g c} \cdot \mathbf{v}_{g}-\bar{\psi}\left(u_{m}^{F}-u_{c}^{F}+\mathbf{v}_{m}\right. \\
& \left.\quad \cdot\left(\mathbf{w}-\mathbf{v}_{m} / 2\right)\right)=-\vartheta
\end{aligned}
$$


where

$\vartheta=T\left(\bar{\rho}_{s} \frac{\partial \eta_{s}}{\partial t}+\bar{\rho}_{c} \frac{\partial \eta_{c}}{\partial t}+\bar{\rho}_{m} \frac{D \eta_{m}}{D t}+\bar{\rho}_{g} \frac{D_{g} \eta_{g}}{D t}-\bar{\psi}\left(\eta_{m}-\eta_{c}\right)\right)$

is a sink of mechanical energy in the medium during the mechanical processes. In other words, it defines the rate of local production of heat in the medium caused by dissipation processes. The last term in (41) represent heat production in wetting processes.

Taking into account that local heat production $\vartheta$ caused by dissipation processes is directly related to local entropy production in the system, according to the second law of thermodynamics we assume that the quantity $\vartheta$ is positive definite

$\vartheta \geq 0$.

From (40) we obtain the inequality

$$
\begin{aligned}
- & \bar{\rho}_{c} \frac{\partial u_{c}^{F}}{\partial t}-\bar{\rho}_{m} \frac{D_{m} u_{m}^{F}}{D t}-\bar{\rho}_{g} \frac{D_{g} u_{g}^{F}}{D t}+\operatorname{tr}\left(\mathbf{D}_{m} \overline{\mathrm{T}}_{m}\right) \\
& +\operatorname{tr}\left(\mathbf{D}_{g} \overline{\mathrm{T}}_{g}\right)-\overline{\boldsymbol{\pi}}_{m c} \cdot \mathbf{v}_{m}-\overline{\boldsymbol{\pi}}_{g c} \cdot \mathbf{v}_{g} \\
& +\bar{\psi}\left(u_{m}^{F}-u_{c}^{F}+\mathbf{v}_{m} \cdot\left(\mathbf{w}-\mathbf{v}_{m} / 2\right)\right) \geq 0
\end{aligned}
$$

which has to be satisfied for all mechanical processes in the unsaturated porous material. Inequality (43) will be called the dissipation inequality of mechanical energy. This inequality is a mechanical version of the second law of thermodynamics. It corresponds to the physical observation that materials at uniform temperature, free from sources of heat, may dissipate the mechanical energy but cannot produce it, [68].

The Helmholtz free energy densities $u_{c}^{F}, u_{m}^{F}$ and $u_{g}^{F}$ are constitutive quantities and should be included into the set (37).

\section{Constitutive equations}

We use the dissipation inequality of mechanical energy (43) to formulate a consistent set of constitutive equations for the proposed model of the capillary transport of liquid and gas in an unsaturated porous medium. This will be done by application of a similar procedure to that used in phenomenological thermodynamics, based on the entropy inequality and the concept of Lagrange multipliers [45, 52, 71, 72].
The dissipation inequality (43) should be satisfied for arbitrary values of the independent quantities (36) and their derivatives describing mechanical processes in the unsaturated porous materials. Some of these quantities are explicitly present in the inequality and the others define the constitutive quantities describing physical properties of the constituents of the medium and their interactions. These fields are related through the mass balance Eqs. (24), (26) and (27), evolution Eq. (28), linear momentum balance Eqs. (31), (33) and (34) and the identity (5). Therefore, dissipation inequality imposes restrictions on the form of the constitutive relations, and the balance equations impose constraints on the independent quantities occurring in that inequality.

The exceptions are linear momentum balance Eqs. (31), (33) and (34). Equation (31), due to immobility of the capillary liquid, defines the volume force $\overline{\boldsymbol{\pi}}_{c s}$ of its interaction with the skeleton, which is not present in the dissipation inequality (43). The momentum balance Eqs. (33) and (34), in turn, do not constrain constitutive relations in (43) because the constitutive quantities in this inequality cannot depend on accelerations. In this case, accelerations are present only in the momentum balance equations, and therefore, their Lagrange'e multipliers must be equal to zero to allow the dissipation inequality to be satisfied (see e.g. [4, 46, 72]).

The number of constraints can be additionally reduced if the identity (5) and Eq. (24) are used as definitions (for example) of the saturation $s_{g}$ and the mass source $\bar{\psi}$, respectively. Such procedure is acceptable because elimination of one quantity from the considered system, linked by the constraint, is an alternative approach to the method of Lagrange multipliers. This enables elimination of both quantities from balance equations and the dissipation inequality.

The use of the mass balance Eq. (24) as a constitutive relation for the mass source of the mobile liquid requires a formulation of the other equation relating independent quantities (36) to provide the system of equations complete. Such equation will be obtained from the dissipation inequality as a condition of the thermodynamic equilibrium of the capillary and the mobile liquids.

Taking scalar functions $A, B$ and $C$ as Lagrange multipliers for the constraints (26), (27), and (28), 
respectively, the inequality (43) can be written in the form

$$
\begin{aligned}
& A\left(\frac{\partial \bar{\rho}_{c}}{\partial t}+\frac{D_{m} \bar{\rho}_{m}}{D t}+\bar{\rho}_{m} \operatorname{div}\left(\mathbf{v}_{m}\right)\right)+B\left(\frac{D_{g} \bar{\rho}_{g}}{D t}+\bar{\rho}_{g} \operatorname{div}\left(\mathbf{v}_{g}\right)\right) \\
& +C f_{v}\left(\frac{\partial^{T} s_{c}}{\partial t}+\frac{\partial^{T} s_{m}}{\partial t}+\operatorname{div}\left(s_{m} \frac{\partial r}{\partial t} \mathbf{v}_{r}\right)-\psi_{m c}^{\mathrm{v}}\right) \\
& -\bar{\rho}_{c} \frac{\partial u_{c}^{F}}{\partial t}-\bar{\rho}_{m} \frac{D_{m} u_{m}^{F}}{D t}-\bar{\rho}_{g} \frac{D_{g} u_{g}^{F}}{D t}+\operatorname{tr}\left(\mathbf{D}_{m} \overline{\mathrm{T}}_{m}\right)+\operatorname{tr}\left(\mathbf{D}_{g} \overline{\mathrm{T}}_{g}\right) \\
& -\overline{\boldsymbol{\pi}}_{m c} \cdot \mathbf{v}_{m}-\overline{\boldsymbol{\pi}}_{g c} \cdot \mathbf{v}_{g}+\frac{\partial \bar{\rho}_{c}}{\partial t}\left(u_{m}^{F}-u_{c}^{F}+\mathbf{v}_{m}\right. \\
& \left.\cdot\left(\mathbf{w}-\mathbf{v}_{m} / 2\right)\right) \geq 0 .
\end{aligned}
$$

The multipliers $A, B$ and $C$ are additional constitutive quantities the functional forms of which have to be determined from the inequality (44).

Taking into account that the physical constituents of porous material filled with liquid and gas remain separated during mechanical processes occurring in this medium, we assume the unsaturated porous material to be a mixture of immiscible constituents [6]. This means that each constituent in the mixture should obey the constitutive relations for that constituent alone. Therefore, it is reasonable to define the Helmholtz free energy density and stress state for each physical constituent independently by quantities describing its own state of deformation.

We assume that all three constituents of the unsaturated porous medium: the mobile liquid, the capillary liquid and the gas, are simple fluids of differential type, [55, 68]. In this case, the local state of deformation for each fluid constituent is defined by its phase density of mass and by gradient of its deformation rate. Then, the Helmholtz free energy density of each constituent in the medium is a unique function of its mass density,

$u_{m}^{F}=u_{m}^{F}\left(\rho_{m}\right), \quad u_{c}^{F}=u_{c}^{F}\left(\rho_{c}\right), \quad u_{g}^{F}=u_{g}^{F}\left(\rho_{g}\right)$,

and gradients of deformation rates allows for the description of viscous dissipation of mechanical energy caused by internal friction in fluids.

Considering relations (45) and the identity (5), the inequality (44) can be written in the form

$$
\begin{aligned}
& f_{v} s_{m} \frac{D_{m} \rho_{m}}{D t}\left(A-\frac{p_{m}}{\rho_{m}}\right)+f_{v} s_{g} \frac{D_{g} \rho_{g}}{D t}\left(B-\frac{p_{g}}{\rho_{g}}\right) \\
& +f_{v} s_{c} \frac{\partial \rho_{c}}{\partial t}\left(A+u_{m}^{F}-u_{c}^{F}-\frac{p_{c}}{\rho_{c}}\right)+\frac{\partial \bar{\rho}_{c}}{\partial t} \mathbf{v}_{m} \cdot\left(\mathbf{w}-\mathbf{v}_{m} / 2\right) \\
& +f_{v} \frac{\partial^{T} s_{m}}{\partial t}\left(A \rho_{m}-B \rho_{g}+C\right)+f_{v} \frac{\partial^{T} s_{c}}{\partial t}\left(\left(A+u_{m}^{F}-u_{c}^{F}\right) \rho_{c}\right. \\
& \left.-B \rho_{g}+C\right)+f_{v}\left(s_{m} d i v\left(\frac{\partial r}{\partial t} \mathbf{v}_{r}\right)-\psi_{m c}^{v}\right) \\
& \quad \times C+f_{v} \frac{\partial r}{\partial t} \mathbf{v}_{r} \cdot \operatorname{grad}^{T}\left(s_{m}\right) C+\operatorname{tr}\left(\mathbf{D}_{m}\left(\overline{\mathrm{T}}_{m}+A \bar{\rho}_{m} \mathbf{I}\right)\right) \\
& +\operatorname{tr}\left(\mathbf{D}_{g}\left(\overline{\mathrm{T}}_{g}+B \bar{\rho}_{g} \mathbf{I}\right)\right)-\mathbf{v}_{m} \cdot\left(\bar{\pi}_{m c}-A f_{v} \rho_{m} \mathbf{g r a d}^{T}\left(s_{m}\right)\right) \\
& -\mathbf{v}_{g} \cdot\left(\bar{\pi}_{g c}-B f_{v} \rho_{g} \mathbf{g r a d}^{T}\left(s_{g}\right)\right) \geq 0,
\end{aligned}
$$

where

$\operatorname{grad}^{T}\left(s_{\alpha}\right) \equiv \operatorname{grad}\left(s_{\alpha}\right)+\frac{\partial s_{\alpha}}{\partial r} \operatorname{grad}(r)$,

for $\alpha=m, g$

and

$p_{m}=\rho_{m}^{2} \frac{d u_{m}^{F}}{d \rho_{m}}, \quad p_{c}=\rho_{c}^{2} \frac{d u_{c}^{F}}{d \rho_{c}}, \quad p_{g}=\rho_{g}^{2} \frac{d u_{g}^{F}}{d \rho_{g}}$

are thermodynamic definitions of pressures in particular constituents. The pressure $p_{c}$ in the capillary liquid, due to its liquid-like character, should be understood as the negative value of the spherical part of the phase stress tensor in that constituent. For its partial stress tensor $\overline{\mathrm{T}}_{c}$, we take the representation

$\overline{\mathrm{T}}_{c}=-f_{v} s_{c} p_{c} \mathbf{I}$.

The dissipation inequality (46) has to be satisfied for any process of the capillary transport of liquid and gas in the unsaturated porous material described by independent fields (36) and their derivatives. Each component on the left-hand side of this inequality may describe some mechanism of the mechanical energy dissipation depending on the assumed constitutive model of the medium. Each component contains at least one constitutive quantity and one independent quantity, and their equipresence indicates the relationship of both quantities in the processes of dissipation. The inequality (46) imposes restrictions on the form of these relations. 
6.1 Constitutive relations for Lagrange multipliers

In the constitutive model of the medium, we assume that changes of mass densities of the constituents of the medium and mass exchange between the capillary and the mobile liquid do not cause dissipation of the mechanical energy. This means that no one constitutive quantity occurring in the inequality (46) is a function of derivatives:

$\frac{D_{m} \rho_{m}}{D t}, \quad \frac{D_{g} \rho_{g}}{D t}, \quad \frac{\partial \rho_{c}}{\partial t}, \quad \frac{\partial \bar{\rho}_{c}}{\partial t} \mathbf{v}_{m}$.

In such case, the left-hand side of the inequality (46) is a linear function of quantities (49). Therefore, the inequality will be satisfied for any values of quantities (49) if the expressions in the brackets at these quantities are identically equal to zero. We obtain

$A \equiv p_{m} / \rho_{m}, \quad B \equiv p_{g} / \rho_{g}, \quad \mathbf{w} \equiv \mathbf{v}_{m} / 2$,

and

$u_{m}^{F}+p_{m} / \rho_{m}=u_{c}^{F}+p_{c} / \rho_{c}$.

Identities (50) define Lagrange multipliers $A, B$ and velocity $\mathbf{w}$, whereas the equality (51) constitute the condition of the thermodynamic equilibrium between the mobile and the capillary liquids. It states that chemical potentials [10,40] of both liquids at the thermodynamic equilibrium are equal. This means that there is no phase transition on the contact surface of both liquids. Such transition can exist, however, in the vicinity of menisci during their motion in the pore space.

Due to the constitutive assumptions (45) and definitions (47), from the equality (51) results that mass densities of the mobile and capillary liquids are uniquely related. This equality replaces the mass balance Eq. (24) in the set of equations relating the independent quantities (36), which has been used as a constitutive equation defining the mass source of the mobile liquid resulting from the mass exchange with the capillary liquid.

Considering constitutive relations (50) and equality (51), the inequality (46) reduces to the form

$$
\begin{aligned}
& f_{v} \frac{\partial^{T} s_{m}}{\partial t}\left(p_{m}-p_{g}+C\right)+f_{v} \frac{\partial^{T} s_{c}}{\partial t}\left(p_{c}-p_{g}+C\right) \\
& \quad+f_{v}\left(s_{m} \operatorname{div}\left(\frac{\partial r}{\partial t} \mathbf{v}_{r}\right)-\psi_{m c}^{v}\right) C+f_{v} \frac{\partial r}{\partial t} \mathbf{v}_{r} \cdot \operatorname{grad}^{T}\left(s_{m}\right) C \\
& +\operatorname{tr}\left(\mathbf{D}_{m}\left(\overline{\mathrm{T}}_{m}+f_{v} s_{m} p_{m} \mathbf{I}\right)\right)+\operatorname{tr}\left(\mathbf{D}_{g}\left(\overline{\mathrm{T}}_{g}+f_{v} s_{g} p_{g} \mathbf{I}\right)\right) \\
& -\mathbf{v}_{m} \cdot\left(\overline{\boldsymbol{\pi}}_{m c}-f_{v} p_{m} \mathbf{g r a d}^{T}\left(s_{m}\right)\right)-\mathbf{v}_{g} \\
& \cdot\left(\overline{\boldsymbol{\pi}}_{g c}-f_{v} p_{g} \operatorname{grad}^{T}\left(s_{g}\right)\right) \geq 0 .
\end{aligned}
$$

The inequality (52) should determine the form of Lagrange multiplier $C$. It is present in the first four components of this inequality related with quantities characterizing changes of the saturation of the medium with the mobile and capillary liquid. These components describe the dissipation of mechanical energy of the medium in processes induced by changes of saturation, e.g. in heat production occurring in wetting processes of the skeleton.

We assume that no one constitutive quantity in the inequality (52) depends on derivatives:

$\partial^{T} s_{m} / \partial t, \quad \partial^{T} s_{c} / \partial t$

In such case, fulfillment of the inequality (52) requires the expressions in brackets occurring at derivatives (53) to be equal to zero. This leads, however, to no unique definition of the Lagrange multiplier $C$. To avoid this contradiction, we assume that saturation of the medium with the capillary liquid is a unique function of saturation with the mobile liquid:

$s_{c}=s_{c}\left(s_{m}\right)$.

Then, due to the identity (5), saturation of the medium with the gas is also unique function of the saturation with the mobile liquid.

Constitutive relation (54) is obvious for processes occurring in porous media of simple capillary structure of the pore space, schematically shown in Fig. 1b-d. It eliminates saturation parameter $s_{c}$ with the capillary liquid from the set (36) of the independent quantities.

Due to relation (54), the derivative $\partial^{T} s_{c} / \partial t$ can be replaced by the derivative $\partial^{T} s_{m} / \partial t$. Then, the inequal- 
ity (52) will be satisfied for any values of the derivative $\partial^{T} s_{m} / \partial t$ if the expression at this derivative is equal to zero. We obtain the following expression defining the Lagrange multiplier $C$,

$C=\frac{d s_{m}}{d s_{g}}\left(p_{m}-p_{g}+\frac{d s_{c}}{d s_{m}}\left(p_{c}-p_{g}\right)\right)$.

Therefore, the inequality (52) reduces to the form

$$
\begin{aligned}
& \frac{d s_{m}}{d s_{g}}\left(p_{m}-p_{g}+\frac{d s_{c}}{d s_{m}}\left(p_{c}-p_{g}\right)\right) \frac{\partial r}{\partial t} \mathbf{v}_{r} \cdot \operatorname{grad}^{T}\left(s_{m}\right) \\
& +f_{v}\left(s_{m} d i v\left(\frac{\partial r}{\partial t} \mathbf{v}_{r}\right)-\psi \psi_{m c}^{\mathrm{v}}\right) \\
& \times \frac{d s_{m}}{d s_{g}}\left(p_{m}-p_{g}+\frac{d s_{c}}{d s_{m}}\left(p_{c}-p_{g}\right)\right) \\
& +\operatorname{tr}\left(\mathbf{D}_{m}\left(\overline{\mathbf{T}}_{m}+f_{v} s_{m} p_{m} \mathbf{I}\right)\right)+\operatorname{tr}\left(\mathbf{D}_{g}\left(\overline{\mathbf{T}}_{g}+f_{v} s_{g} p_{g} \mathbf{I}\right)\right) \\
& -\mathbf{v}_{m} \cdot\left(\bar{\pi}_{m c}-f_{v} p_{m} \mathbf{g r a d}^{T}\left(s_{m}\right)\right) \\
& -\mathbf{v}_{g} \cdot\left(\bar{\pi}_{g c}-f_{v} p_{g} \operatorname{grad}^{T}\left(s_{g}\right)\right) \geq 0 .
\end{aligned}
$$

\subsection{Constitutive relations for quantities describing menisci motion}

Two first components in the inequality (56) are related with quantities describing menisci motion in the pore space and characterize the dissipation processes occurring in the medium during changes of its saturation with the constituents. The constitutive model of these processes should provide both components of the inequality (56) to be positive definite. This impose restrictions on the functional form of constitutive relations for three present there constitutive quantities: $\mathbf{v}_{r}, \partial r / \partial t$ and $\psi_{m c}^{\mathrm{v}}$.

Constitutive relation for the "velocity" $\mathbf{v}_{r}$ should define the mechanism of menisci motion in the skeleton pore space induced by changes of the quantity $r$. In the model developed here, we assume a diffusion mechanism of the menisci motion in the pressurespace continuum, analogous to the diffusion motion of molecules in the time-space continuum. For successive values of the parameter $r$, the menisci move stepwise from one position to another, just like during molecule diffusion recorded at successive moments in time. It is assumed, therefore, that the part of the mass flux of the mobile liquid and the gas induced by menisci motion is proportional to the gradient of saturation with the mobile liquid. We propose
$\mathbf{v}_{r}=-C_{m}\left(s_{m}, r\right) \operatorname{grad}^{T}\left(s_{m}\right)$.

The gradient of saturation with the mobile liquid may be considered as a local measure of the menisci surface which determines the mass flux of the mobile liquid and gas transported with the menisci. The coefficient $C_{m}\left(s_{m}, r\right)$ in relation (57) is positive and should be determined experimentally. The minus sign in this equation reflects the fact that with an increase in the quantity $r$, the direction of menisci motion is opposite to that of the gradient of saturation with the mobile liquid.

The constitutive relation (57) is an analogue of Fick's first law of diffusion. The important difference between the process of molecule diffusion and the quasi-static motion of liquid in the unsaturated porous material is that the first occurs in time-space continuum in which diffusion processes are unidirectional, determined by the direction of changes in time, while the second occurs in pressure-space continuum, where both directions of pressure changes are possible.

A similar relation can be formulated, taking the gradient of saturation $s_{g}$ with the gas as a local measure of the menisci surface. It takes the form

$\mathbf{v}_{r}=C_{g}\left(s_{g}, r\right) \operatorname{grad}^{T}\left(s_{g}\right)$.

However, due to (54) and the identity (5) we have

$\operatorname{grad}^{T}\left(s_{g}\right)=d s_{g} / d s_{m} \operatorname{grad}^{T}\left(s_{m}\right)$.

This means that both constitutive Eqs. (57) and (58) will be identical if the coefficients $C_{m}\left(s_{m}, r\right)$ and $C_{g}\left(s_{g}, r\right)$ satisfy the relation

$C_{g}\left(s_{g}, r\right)=-d s_{g} / d s_{m} C_{m}\left(s_{m}, r\right)$.

On account of the analogy between constitutive relation (57) and that in the diffusion theory, this relation will be called diffusion law of capillary transport of menisci in unsaturated porous materials.

Considering the constitutive relation (57) and the fact that the quantity $d s_{m} / d s_{g}$ takes negative values for any processes in the unsaturated porous materials, the first component in the inequality (56) will be positively definite if the product

$$
\left(p_{m}-p_{g}-\frac{d s_{c}}{d s_{m}}\left(p_{c}-s_{g}\right)\right) \frac{\partial r}{\partial t}
$$

is positive. This means that the constitutive quantity $\partial r / \partial t$ has to be a function of the expression occurring 
in the bracket of product (61). For the linear case we have

$\frac{\partial r}{\partial t}=C_{r}\left(s_{m}, r\right)\left(p_{m}-p_{g}-\frac{d s_{c}}{d s_{m}}\left(p_{c}-p_{g}\right)\right)$

where $C_{r}\left(s_{m}, r\right)$ is positive coefficient characterising non-stationary processes of menisci transport in porous materials. This coefficient should be experimentally determined.

Constitutive Eq. (62) defines evolution of the parameter $r$. Equation (62), written for stationary processes in the form

$d s_{m}\left(p_{m}-p_{g}\right)+d s_{c}\left(p_{c}-p_{g}\right)=0$,

can be interpreted as a balance equation of energy exchanged by the capillary and mobile liquids during the mass exchange between them. It shows that the sum of the work done by both liquids during the mass exchange is equal to zero. Therefore, the constitutive Eq. (62) can be treated as the general form of this balance, accounting for energy dissipation during mass exchange in no stationary processes.

Considering the constitutive relation (62) and that the quantity $d s_{m} / d s_{g}$ takes negative values, the second component in the inequality (56) will be positively definite if the expression in bracket containing the constitutive quantity $\psi_{m c}^{\mathrm{v}}$ is a linear function of the derivative $\partial r / \partial t$. This allows for the formulation of the constitutive relation for the quantity $\psi_{m c}^{\mathrm{v}}$. We have

$\psi_{m c}^{\mathrm{v}}=s_{m} \operatorname{div}\left(\frac{\partial r}{\partial t} \mathbf{v}_{r}\right)+C_{s}\left(s_{m}, r\right) \frac{\partial r}{\partial t}$

where $C_{s}\left(s_{m}, r\right)$ is a positive coefficient characterising changes of saturation of the pore space with the mobile liquid caused by the volumetric motion of menisci. The coefficient $C_{s}\left(s_{m}, r\right)$ should be experimentally determined.

Equation (63) has a number of interesting consequences. Taking into account that in the quasi-static processes of non-wetting liquid transport in unsaturated porous materials (Fig. 1b) both quantities - the capillary pressure $\hat{p}_{c}=p_{m}-p_{g}$ and the ratio $d s_{c} / d s_{m}$ of saturation increments of the mobile and the capillary liquids—-take positive values:

$\hat{p}_{c}>0, \quad d s_{c} / d s_{m}>0$,

equality (63) implies that the relative pressure $p_{c}-p_{g}$ in the capillary liquid in such processes is always negative. Even when there is no gas in the medium, the pressure $p_{c}$ in the capillary liquid is negative. This means that this constituent cannot be treated as an ordinary liquid. Due to the negative pressure in the capillary liquid and its immobility resulting from direct contact with the skeleton, it should be regarded as a solid-like constituent.

Since the intrusion of non-wetting liquid into porous material requires the application of external forces, we will assume that the Helmholtz free energy of a non-wetting capillary liquid is greater than that of the mobile liquid, which we identify with the liquid outside the porous medium,

$u_{c}^{F}>u_{m}^{F}$.

In porous materials containing wetting liquid we have to distinguish two processes: saturation of a dry skeleton and liquid motion in a wet skeleton. In the first case (Fig. 1c) the capillary liquid forms a film on the surface of saturated pores, and its Helmholtz free energy is smaller than that of the mobile liquid,

$u_{m}^{F}>u_{c}^{F}$.

Therefore, the processes of saturation of the medium with wetting liquid, when the film of the capillary liquid is produced, are running spontaneously. Then, a part of the energy difference $\Delta u^{F}=$ $u_{m}^{F}-u_{c}^{F}$ is transformed into heat, called wetting heat, [29], and the rest is used up as a work down on the extruded gas. In this case, the ratio $d s_{c} / d s_{g}$ of saturation increments is negative, and from (51) as well as (63) we obtain

$$
\begin{gathered}
p_{c}-\frac{\rho_{c}}{\rho_{m}} p_{m}=\rho_{c}\left(u_{m}^{F}-u_{c}^{F}\right)>0, \\
p_{m}-p_{g}=\left(p_{c}-p_{m}\right) \frac{d s_{c}}{d s_{g}}<0 .
\end{gathered}
$$

Since the mass densities $\rho_{c}, \rho_{m}$ satisfy the inequality $\rho_{c}>\rho_{m}$, and the pressure in the mobile liquid is always positive, the inequality $(66)_{1}$ shows that the pressure in the wetting capillary liquid in this case must also be positive. In turn, the inequality $(66)_{2}$ shows that the necessary condition for the local static equilibrium of the system is the pressure in the gas to be greater than the pressure in the mobile liquid.

In the second case (Fig. 1d), when liquid moves in the wet skeleton, the amount of the capillary liquid contained in the film on the skeleton surface does not 
change, however, a new film containing this liquid appears in unsaturated pores that covers the wetted surface of the skeleton. In this case, production of the film of the capillary liquid takes place during the extrusion process and needs the external work. Therefore, its Helmholtz free energy is greater than that of the mobile liquid,

$u_{c}^{F}>u_{m}^{F}$.

Nevertheless, the saturation of the wetted porous materials is running spontaneously, driven by the energy of the capillary liquid. Part of the energy difference $\Delta u^{F}=u_{c}^{F}-u_{m}^{F}$ is transformed into heat (wetting heat) and the rest is used up as a work down on the extruded gas. Taking into account that in this case, the ratio $d s_{c} / d s_{m}$ of saturation increments and the capillary pressure $\hat{p}_{c}=p_{m}-p_{g}$ are always negative,

$d s_{c} / d s_{m}<0, \quad \hat{p}_{c}=p_{m}-p_{g}<0$,

from (63) it results that the relative pressure in the wetting capillary liquid in unsaturated pores is negative also,

$p_{c}-p_{g}<0$.

This can explain the apparent cohesion in unsaturated soils observed in laboratory tests [35], or in numerical simulations of unsaturated granular media $[65,66]$.

\subsection{Constitutive relations for stresses and the interaction forces}

Due to the independence of the quantities:

$\mathbf{D}_{m}, \quad \mathbf{D}_{g}, \quad \mathbf{v}_{m}, \quad \mathbf{v}_{g}$,

inequality (56) will be satisfied for any changes of these quantities if each term in which they are contained is positively definite or equal to zero.

Making the constitutive assumption that the stress tensors $\overline{\mathbf{T}}_{m}$ and $\overline{\mathbf{T}}_{g}$ in the mobile liquid and gas are functions of their strain rate tensors $\mathbf{D}_{m}, \mathbf{D}_{g}$, for the expressions appearing in brackets next to those tensors we obtain the relations

$$
\begin{gathered}
\overline{\mathbf{T}}_{m}=-f_{v} s_{m} p_{m} \mathbf{I}+\overline{\boldsymbol{\sigma}}_{m}\left(\mathbf{D}_{m}\right), \\
\overline{\mathbf{T}}_{g}=-f_{v} s_{g} p_{g} \mathbf{I}+\overline{\boldsymbol{\sigma}}_{g}\left(\mathbf{D}_{g}\right),
\end{gathered}
$$

where the functions $\overline{\boldsymbol{\sigma}}_{m}\left(\mathbf{D}_{m}\right)$ and $\overline{\boldsymbol{\sigma}}_{g}\left(\mathbf{D}_{g}\right)$ can be interpreted as partial viscous stress tensors in the constituents. These functions have to satisfy the conditions

$\operatorname{tr}\left(\mathbf{D}_{m} \overline{\boldsymbol{\sigma}}_{m}\left(\mathbf{D}_{m}\right)\right) \geq 0, \quad \operatorname{tr}\left(\mathbf{D}_{g} \overline{\boldsymbol{\sigma}}_{g}\left(\mathbf{D}_{g}\right)\right) \geq 0$,

to ensure that the energy dissipated by internal viscous interactions in both constituents is positive definite. In the case of linear dependence between the quantities $\overline{\boldsymbol{\sigma}}_{m}, \overline{\boldsymbol{\sigma}}_{g}$ and $\mathbf{D}_{m}, \mathbf{D}_{g}$, respectively, the constitutive relations (69) take the form

$\overline{\mathbf{T}}_{m}=-f_{v} s_{m} p_{m} \mathbf{I}+\eta_{m} \mathbf{D}_{m}, \quad \overline{\mathbf{T}}_{g}=-f_{v} s_{g} p_{g} \mathbf{I}+\eta_{g} \mathbf{D}_{g}$

Then, the inequalities in (70) imply that the coefficients $\eta_{m}, \eta_{g}$ have to be positive:

$\eta_{m}>0, \quad \eta_{g}>0$.

The viscous stresses are often omitted in internal regions of fluid flow through porous materials, as they are very small in comparison with the force of viscous interaction with the skeleton. However, they have to be taken into account when the fluid is flowing along the boundary of a porous material, where the fluid strain rate is the greatest $[11,43]$.

In turn, making the constitutive assumption that the viscous interaction forces $\overline{\boldsymbol{\pi}}_{m c}$ and $\overline{\boldsymbol{\pi}}_{g c}$ of the mobile liquid and gas with the capillary liquid are functions of their relative velocities $\mathbf{v}_{m}$ and $\mathbf{v}_{g}$, respectively, for the expressions in inequality (56) appearing in brackets next to these velocities we obtain the relations,

$\bar{\pi}_{m c}=f_{v} p_{m} \operatorname{grad}^{T}\left(s_{m}\right)+\overline{\mathbf{R}}_{m}\left(\mathbf{v}_{m}\right)$,
$\bar{\pi}_{g c}=f_{v} p_{g} \operatorname{grad}^{T}\left(s_{g}\right)+\overline{\mathbf{R}}_{g}\left(\mathbf{v}_{g}\right)$.

The last terms in (73) represent forces of viscous interactions of the constituents resulting from their relative motion. Due to the assumed isotropy of the pore space structure, they reduce to the form

$\overline{\mathbf{R}}_{\alpha}\left(\mathbf{v}_{\alpha}\right)=-\bar{R}_{\alpha}\left(\mathbf{v}_{\alpha}\right) \mathbf{v}_{\alpha} \quad(\alpha=m, g)$,

where $\bar{R}_{m}\left(\mathbf{v}_{m}\right)$ and $\bar{R}_{g}\left(\mathbf{v}_{g}\right)$ are the coefficients of flow resistance of the mobile liquid and gas through the unsaturated porous medium.

The dissipation inequality (59) will be satisfied for any values of velocities $\mathbf{v}_{m}$ and $\mathbf{v}_{g}$ if the functions $\overline{\mathbf{R}}_{m}\left(\mathbf{v}_{m}\right), \overline{\mathbf{R}}_{g}\left(\mathbf{v}_{g}\right)$ satisfy the inequalities 
$\mathbf{v}_{m} \cdot \overline{\mathbf{R}}_{m}\left(\mathbf{v}_{m}\right) \leq 0, \quad \mathbf{v}_{g} \cdot \overline{\mathbf{R}}_{g}\left(\mathbf{v}_{g}\right) \leq 0$.

This means that the coefficients $R_{m}\left(\mathbf{v}_{m}\right)$ and $R_{g}\left(\mathbf{v}_{g}\right)$ in representations (74) have to be positive.

For processes in which the gas is in direct contact with the skeleton (Fig. 1c), the volume interaction farce $\bar{\pi}_{g c}$ should be replaced by the force $\bar{\pi}_{g s}$. It can be shown that in this case the constitutive relation for $\bar{\pi}_{g s}$ takes the form

$\bar{\pi}_{g s}=p_{g} \operatorname{grad}\left(s_{g} f_{v}\right)+\overline{\mathbf{R}}_{g}\left(\mathbf{v}_{g}\right)$,

where as the constitutive relation for $\overline{\boldsymbol{\pi}}_{m c}$ remains the same, given by 73$)_{1}$.

The force $\overline{\mathbf{R}}_{g}\left(\mathbf{v}_{g}\right)$ of viscous interaction between constituents has the representation

$\overline{\mathbf{R}}_{g}\left(\mathbf{v}_{g}\right)=-\bar{R}_{g}\left(\mathbf{v}_{g}\right) \mathbf{v}_{g}$,

where $\bar{R}_{g}\left(\mathbf{v}_{g}\right)$ is positively definite coefficient of flow resistance.

The forms of constitutive relations (69) or (71) and (73) or (76) for stress tensors in the constituents and for volume interaction forces, respectively, are wellknown in the literature. However, they were derived for constituents of unsaturated porous materials defined in a different way (e.g. [32]) or in the mixture theory, e.g. of multicomponent particulate flows [49].

Constitutive relations (45), (47), (48), (50), (54), (57), (62), (64), (69), (73), formulated in this section, together with the balance Eqs. (24) and (31) (used as definitions of the constitutive quantities $\psi$ and $\overline{\boldsymbol{\pi}}_{c s}$ ), form the system of 45 scalar equations for 45 (scalar) constitutive quantities:

$$
\begin{aligned}
& u_{m}^{m}, u_{c}^{m}, u_{g}^{m}, p_{m}, p_{c}, p_{g}, \overline{\mathbf{T}}_{c}, \mathbf{w}, s_{c}, \mathbf{v}_{r}, r, \\
& \quad \overline{\mathbf{T}}_{m}, \overline{\mathbf{T}}_{g}, \overline{\boldsymbol{\pi}}_{c m}, \overline{\boldsymbol{\pi}}_{c g}, \psi, \overline{\boldsymbol{\pi}}_{c s} .
\end{aligned}
$$

describing mechanical properties of the fluid constituents in the unsaturated porous material and their interactions. These equations complete the balance Eqs. (26)-(28), (33), (34), (51) and the identity (5) (11 equations) defining independent fields (11 scalar quantities):

$\rho_{m}, \rho_{c}, \rho_{g}, s_{m}, s_{g}, \quad \mathbf{v}_{m}, \mathbf{v}_{g}$.

Therefore, the obtained system of equations is closed and enables formulation of boundary value problems for mechanical processes in unsaturated porous materials.

\section{Conclusions and final remarks}

A new macroscopic model of liquid and gas transport in unsaturated porous materials has been presented. The considerations were based on the methods and concepts of multi-constituent continuum mechanics. The key element of the proposed physical model of the unsaturated medium is division of the liquid in the pore space into two constituents: the mobile and the capillary liquid, which exchanges mass and energy during motion of the menisci. The mobile liquid retains the physical properties of the liquid outside the pore space, while the capillary liquid forms a film on the contact surface with the skeleton. The capillary liquid is immobile, contains the whole capillary energy of the system, and-due to possible negative pressure within it-has solid-like properties. The kinematics of the mobile liquid and the gas are described by three velocity fields. Two of them characterise the free motion of each constituent in the regions they currently occupy in the pore space, and the third characterizes the common motion of their parts associated with the motion of menisci. For all fluid constituents of the porous medium, balance equations of mass and linear momentum were derived and evolution equations for saturations were formulated. These equations have been supplemented by constitutive relations for mechanical processes. The approach was used based on the analysis of dissipation inequality of mechanical energy in the medium and on the Lagrange multipliers method. The closed system of equations was obtained, allowing for the formulation of boundary value problems for mechanical processes in unsaturated porous materials.

The mathematical model formulated here gives an alternative description of mechanical processes of liquid and gas transport in unsaturated porous materials. From the equations obtained, it follows that processes of capillary transport in such systems take place in five-dimensional pressure-time-space. Therefore, any attempt to describe them in terms of temporal and spatial changes only must always lead to ambiguous relations and inexplicable effects resulting from the reduction of one dimension of their course space.

It can be shown that the equations obtained describe both quasi-static and quasi-stationary processes of capillary transport of liquid and gas in unsaturated porous materials as special cases of the new 
mathematical model. This concern, among others, the description of quasi-static intrusion of mercury into porous materials and description of liquid or gas flow through unsaturated porous material. In the first case, the equations have a form characteristic for nonstationary processes in which, instead of time as the independent variable, the mercury pressure is present, [15]. In the second case, for example, even the geometrically very simple problem of liquid flow through a layer of unsaturated porous material is described by a system of three coupled, nonlinear equations for distributions of saturation, the fluid pressure and its flow velocity in the layer. In this case the fluid flow through the layer of unsaturated porous material is similar to flow through an elastic tube the cross-section of which is being changed depending on the local pressure in fluid. A detailed description of these phenomena will be the subject of next papers.

Acknowledgments This work was partially supported by the Polish Ministry of Science and Higher Education under the research project No. N N501 325335

Open Access This article is distributed under the terms of the Creative Commons Attribution 4.0 International License (http:// creativecommons.org/licenses/by/4.0/), which permits unrestricted use, distribution, and reproduction in any medium, provided you give appropriate credit to the original author(s) and the source, provide a link to the Creative Commons license, and indicate if changes were made.

\section{References}

1. Aksielrud GA, Altszuler MA (1987) Mass transport in porous materials (in Polish). WNT, Warsaw

2. Andreaus U, Giorgio I, Lekszycki T (2014) A 2-D continuum model of a mixture of bone tissue and bio-resorbable material for simulating mass density redistribution under load slowly variable in time. ZAMM 66(1):209-237

3. Aziz K, Settari A (1979) Petroleum reservoir simulation. Applied Science Publishers, London

4. Bargmann S, Steinmann P (2007) Classical results for a non-classical theory: remarks on thermodynamic relations in Green-Naghdi thermo-hyperelasticity. Contin Mech Thermodyn 19(1-2):59-66

5. Bear J (1972) Dynamics of fluid flow in porous media. Elsevier, New York

6. Bedford DE, Drumheller DS (1983) Theories of immiscible and structured mixtures. Int J Eng Sci 21(8):863-960

7. Bennethum LS, Cushman JH (2002) Multicomponent, multiphase thermodynamics of swelling porous media with electroquasistatics: I. Macroscale field equations. Transp Porous Media 47:337-362
8. Bennethum LS, Cushman JH (2002) Multicomponent, multiphase thermodynamics of swelling porous media with electroquasistatics: II. Constitutive theory. Transp Porous Media 47:337-362

9. Benzoni-Gavage S (2001) Linear stability of propagating phase boundaries in capillary fluids. Phys D 155(3):235273

10. Callen HB (1980) Thermodynamics. Wiley, New York

11. Cieszko M, Kubik J (1999) Derivation of matching conditions at the contact surface between fluid-saturated porous solid and bulk fluid. Transp Porous Media 34:319-336

12. Cieszko M (2000) Mechanics of fluid in anisotropic space. Modelling of fluid motion in porous medium. In: Ehlers W (ed) IUTAM symposium on theoretical and numerical methods in continuum mechanics of porous materials, Kluwer Academic Publishers

13. Cieszko M (2001) Mechanics of fluid in anisotropic pore space of permeable materials. Application of Minkowski Metric Space (in Polish). Publishing House of Kazimierz Wielki University, Bydgoszcz

14. Cieszko M (2009) Description of anisotropic pore space structure of permeable materials based on minkowski metric space. Arch Mech 61(6):425-444

15. Cieszko M, Czapla E, Kempiński M (2015) Continuum description of quasi-static intrusion of non-wetting liquid into a porous body. Contin Mech Thermodyn 27(1):133-144. doi:10.1007/s00161-014-0340-2

16. Coleman BD (1963) On thermodynamics of materials with memory. Arch Ration Mech Anal 13:167-178

17. Collins RE (1961) Flow of fluids through porous materials. Reinhold Pub. Corp, New York

37. dell'Isola F, Darve F, Gouin H, Rotoli G (1996) Nucleation of spherical shell-like interfaces by second gradient theory: Numerical simulations. Eur J Mech B Fluids 15:545-568

38. dell'Isola F, Guarascio M, Hutter K (2000) A variational approach for the deformation of a saturated porous solid. A second-gradient theory extending Terzaghi's effective stress principle. Arch Appl Mech 70(5):323-337

18. Drake IC (1949) Pore size distribution in porous materials. Ind Eng Chem 41(4):780

19. Dullien FAL (1992) Porous media: fluid transport and pore structure. Acad. Press, San Diego

20. Eringen AC (1980) Mechanics of continua. Huntington, New York

21. Gray WG, Hassanizadeh SM (1847) Paradoxes and realities in unsaturated flow theory. Water Resour Res 28(8):1991

22. Gray WG, Miller CT (2005) Thermodynamically constrained averaging theory approach for modeling flow and transport phenomena in porous medium systems: 1. Motivation and overview. Adv Water Resour 28:161-180

23. Gray WG, Miller CT (2006) Thermodynamically constrained averaging theory approach for modeling flow and transport phenomena in porous medium systems: 3 . Singlefluid-phase flow. Adv Water Resour 29:1745-1765

24. Gray WG, Miller CT (2009) Thermodynamically constrained averaging theory approach for modeling flow and transport phenomena in porous medium systems: 5. Singlefluid-phase transport. Adv Water Resour 32:681-711

25. Gray WG, Miller CT (2011) TCAT analysis of capillary pressure in non-equilibrium. Two-fluid-phase porous medium systems. Adv Water Resour 34:770-778 
26. Gray WG, Miller CT, Schrefler BA (2013) Averaging theory for description of environmental problems: What we have learned? Adv Water Resour 51:123-138

27. Grim RE (1953) Clay mineralogy, 1st edn. McGraw-Hill, New York

28. Gouin H (1988) Utilization of the second gradient theory in continuum mechanics to study the motion and thermodynamics of liquid-vapor interfaces. Physicochem Hydrodyn 174:667-682 (NATO ASI Series)

29. Gouin H (1996) The second gradient theory applied to interfaces: models of continuum mechanics for fluid interfaces (Chapter). In: Dynamics of multiphase flows across interfaces, Lecture notes in physics, vol 467, pp 8-13

30. Hassanizadeh SM, Gray WG (1980) General conservation equations for multi-phase systems: 3 . Constitutive theory for porous media flow. Adv Water Resour 3:25-40

31. Hassanizadeh SM, Gray WG (1990) Mechanics and thermodynamics of multiphase flow in porous media including interphase boundaries. Adv Water Resour 13:169-186

32. Hassanizadeh SM, Gray WG (1993) Thermodynamic basis of capillary pressure in porous media. Water Resour Res 29(10):3389-3405

33. Hicher PY (1998) Experimental behavior of granular materials. In: Combou B (ed) Behaviour of granular materials. Springer, New York

34. Hilfer R (2006) Macroscopic capillarity without a constitutive capillary pressure function. Phys A 371:209

35. Hilfer R (1998) Macroscopic equations of motion for two phase flow in porous media. Phys Rev E 58:2090

36. Hilfer R (2000) Macroscopic two phase flow in porous media. Phys B 279:125

39. Jackson AS, Miller CT, Gray WG (2009) Thermodynamically constrained averaging theory approach for modeling flow and transport phenomena in porous medium systems: 6. Two-fluid-phase flow. Adv Water Resour 32:779-795

40. Job G, Herrmann F (2006) Chemical potential-a quantity in search of recognition. Eur J Phys 27(2):353-371. doi:10. 1088/0143-0807/27/2/018

41. Kowalski SJ (2003) Thermomechanics of drying processes. Springer, Berlin

42. Kowalski SJ (2004) Engineering of porous materials. Publishing House of Poznań University of Technology, Poznań (in Polish)

43. Kubik J, Cieszko M (2005) Analysis of matching conditions at the boundary surface of fluid-saturated porous solid and bulk fluid: the use of lagrange multipliers. Contin Mech Thermodyn 17:351

44. Leverett M (1941) Capillary behaviour in porous media. Trans ASME 142:152

45. Liu IS (1972) Method of lagrange multipliers for exploitation of the entropy principle. Arch Ration Mech Anal 46:131

46. Liu IS (2008) Entropy flux relation for viscoelastic bodies. J Elast 90:259-270

47. Madeo A, dell'Isola F, Darve F (2013) A continuum model for deformable, second gradient porous media patialy saturated with compressible fluids. J Mech Phys Solids 61:2196-2211

48. Marsily G (1986) Quantitative hydrology-groundwater hydrology for engineers. Academic Press, San Diego
49. Massoudi M (2003) Constitutive relation for the interaction force in multicomponent particulate flows. Int $\mathrm{J}$ Nonlinear Mech 38:313-336

50. Miller CT, Gray WG (2005) Thermodynamically constrained averaging theory approach for modeling flow and transport phenomena in porous medium systems: 2. Foundation. Adv Water Resour 28:181-202

51. Miller CT, Gray WG (2005) Thermodynamically constrained averaging theory approach for modeling flow and transport phenomena in porous medium systems: 4. Species transport fundamentals. Adv Water Resour 28:181-202

52. Miller I (1985) Thermodynamics. Pitman, Boston

53. Mujumdar AS (ed) (2007) Hadbook of industrial drying, 3rd edn. Taylor \& Francis, London

54. Müller TM, Gurevich B (2004) One-dimensional random saturation model for velocity and attenuation in porous rocks. Geophysics 69(5):1166

55. Noll W (1974) The foundations of mechanics and thermodynamics. Springer, Berlin

56. Norris AN (1993) Low-frequency dispersion and attenuation in partially saturated rocks. JASA 94:359

57. Palubarinova-Kocina PJ (1977) Theory of soil water motion. Science, Moscov (in Russian)

58. Pinder GF, Gray WG (2008) Essentials of multiphase flow and transport in porous media. Wiley, London

59. Placidi L (2014) A variational approach for a nonlinear onedimensional damage-elasto-plastic second-gradient continuum model. Contin Mech Thermodyn. doi:10.1007/s00161014-0405-2

60. Placidi L (2014) A variational approach for a nonlinear 1-dimensional second gradient continuum damage model. Contin Mech Thermodyn. doi:10.1007/s00161-014-0338-9

61. Placidi L, dell'Isola F, Ianiro N, Sciarra G (2008) Variational formulation of pre-stressed solid-fluid mixture theory, with an application to wave phenomena. Eur J Mech A Solids 27(4):582-606

62. Richards LA (1931) Capillary conduction of liquids through porous mediums. Physics 1:318

63. Scerrato D, Giorgio I, Della CA, Madeo A, Limam A (2015) A micro-structural model for dissipation phenomena in the concrete. Int J Numer Anal Meth Geomech. doi:10.1002/ nag. 2394

64. Scheidegger A (1957) The physics of flow through porous media. University of Toronto Press, Canada

65. Scholtes L, Hicher PY, Nicot F, Schareyre B, Darve F (2009) On the capillary stress tensor in wet granular materials. Int J Numer Anal Math Geomech 33:1289

66. Scholtes L, Hicher PY, Nicot F, Darve F (2009) Micromechanics of granular materials with capillary effects. Int $\mathbf{J}$ Eng Sci 47:1460

67. Sciarra G, dell'Isola F, Coussy O (2009) Second gradient poromechanics. Int J Solids Struct 46:3150-3164

68. Truesdell C, Noll W (1965) Encyclopedia of physics. In: Flügge $\mathrm{S}$ (ed) The non-linear field theories of mechanics, vol III/3, sec. 79, Springer, Berlin

69. Volfkovich YM, Bagotzky VS (1994) The method of standard porosimetry: 1. Principles and possibilities. 2. Investigation of the formation of porous structures. J Power Sour 48(3):327-348 
70. Volfkovich YM, Bagotzky VS, Sosenkin VE, Blinov IA (2001) The Standard contact porosimetry. Colloids Surf A 187-188:349

71. Wang Y, Hutter K (1999) A constitutive model of multiphase mixtures and its application in shearing flows of saturated solid-fluid mixture. Granul Metter 1:163
72. Wilmański K (1995) Lagrangean model of two-phase porous material. J Nonequilib Thermodyn 20:50-77

73. Zaradny H (1993) Groundwater flow in saturated and unsaturated soil. A.A. Balkema, Avereest 